\title{
Las actitudes como factor clave en la inclusión universitaria
}

\author{
Attitudes as a key factor for inclusion at university
}

\begin{abstract}
Resumen
Las actitudes pueden facilitar o dificultar el proceso inclusivo en la educación superior. Los objetivos del presente estudio son determinar las actitudes del alumnado y docentes hacia la discapacidad y conocer las variables que influyen en dichas actitudes. Se ha realizado un estudio descriptivo de prevalencia realizado en la Universidade da Coruña (UDC) con docentes ( $n=121$ ) y alumnos/as $(n=345)$ utilizando un cuestionario validado, autoadministrado y anonimizado (CUNIDIS). Se realizó un análisis descriptivo y un ANOVA para determinar las variables asociadas a las diferentes actitudes. Los resultados obtenidos manifiestan que los participantes creen preciso adaptar los materiales utilizados en las aulas, pero no los criterios de evaluación y cualificación. Sin embargo, indican que estas adaptaciones no se llevan a cabo realmente. La mayoría de los docentes indicaron que no están suficientemente formados para dar respuesta a las necesidades de los estudiantes con discapacidad. Se considera, por lo tanto, que los estudiantes y docentes de la UDC presentan, de manera general, unas actitudes favorables hacia la inclusión de los estudiantes con discapacidad.
\end{abstract}

\section{Palabras clave}

Educación superior, discapacidades, actitudes, inclusión.

\begin{abstract}
Attitudes can facilitate or hinder the inclusive process in higher education. The aims of this study are exploring the attitudes of students and teachers towards disability and knowing which variables influence those attitudes. A descriptive prevalence study was carried out at the University of A Coruña (UDC) with teachers $(n=121)$ and students ( $n$ $=345$ ) using a validated, self-administered and anonymous questionnaire (CUNIDIS). A descriptive analysis and an ANOVA were performed to determine the variables associated with the different attitudes. The results obtained show that the participants believe that it is necessary to adapt the materials used in the classrooms, but not the evaluation and qualification criteria. However, they indicate that these adaptations are not really carried out. Most teachers stated that they are not sufficiently trained to respond to the needs of students with disabilities. Therefore, it is considered that the students and teachers of the UDC present, in a general way, favorable attitudes towards the inclusion of students with disabilities.
\end{abstract}

\section{Keywords}

Higher education, disabilities, attitudes, inclusion.

\author{
Jessica Garabal-Barbeira \\ <jessica.garabal@udc.es> \\ Universidad de La Coruña. España

\section{Thais Pousada García} \\ <tpousada@udc.es>
}

Universidad de La Coruña. España

\section{Pablo Clemente Espinosa \\ Breen \\ <pespinosa@udc.es> \\ Universidad de La Coruña. España}

\section{Jesús Luis Saleta Canosa <saleta@udc.es>}

Universidad de La Coruña. España

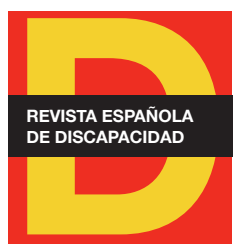

Para citar:

Garabal-Barbeira, J. et al. (2018): "Las actitudes como factor clave en la inclusión universitaria". Revista Española de Discapacidad, 6 (I): 181-198.

Doi: <https://doi.org/10.5569/23405104.06.01.09>

Fecha de recepción: 22-09-2017 Fecha de aceptación: 11-04-2018

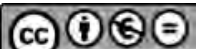




\section{Introducción}

La educación es un fenómeno universal para la inclusión social y la participación de toda persona, es por ello un área fundamental para las personas con discapacidad. Debido a esto, asegurar un sistema escolar lo más inclusivo posible debe ser uno de los principales pilares de toda política de discapacidad. No sólo es importante para los estudiantes con diversidad funcional poder estar con otros estudiantes, sino que para las personas sin esta condición es también un aprendizaje fundamental de la discapacidad como un elemento más de la diversidad de su sociedad (De Luna, 2004).

Según la Clasificación Internacional del Funcionamiento, de la Discapacidad y de la Salud (CIF), la discapacidad "es un término genérico que incluye déficits, limitaciones en la actividad y restricciones en la participación, e indica los aspectos negativos de la interacción entre un individuo y sus factores contextuales" (Organización Mundial de la Salud, 2001). El fenómeno de la discapacidad tiene múltiples dimensiones y afecta a muchos ámbitos en los que se desarrolla la vida diaria de las personas. En conjunto, puede repercutir a la educación de los niños desde las edades más tempranas, por ello, ha de seguir siendo el objetivo principal una buena inclusión en la escuela. Las cifras recogidas en una publicación del Eurostat (Cambois, 2001) indican que las personas con discapacidad presentan menores niveles de acceso a la educación superior, prueba de esto es que el $18 \%$ de las personas sin discapacidad completaron estudios de tercer ciclo frente al $9 \%$ de las personas con discapacidad. Este porcentaje en España es inferior, llegando a hablar únicamente de un $4 \%$ de personas con discapacidad que alcanzan estudios de tercer ciclo (De Luna, 2004).

Las personas con discapacidad ven limitadas sus oportunidades de ingresar en la escuela, permanecer en ella y superar los cursos sucesivos (Fundación Universia y CERMI, 2013). En un mundo cada vez más diverso, el sistema educativo debe comprometerse a ofrecer una respuesta educativa para todo el alumnado, independientemente de sus características individuales. No obstante, aun cuando en el discurso nadie parece desconocer el valor de la inclusión en la sociedad en general, no se logran satisfacer las necesidades educativas del colectivo de personas con discapacidad (Gómez e Infante, 2004). La mejora significativa del nivel medio de los estudios alcanzados para las personas con discapacidad no ha logrado evitar que la exclusión de la educación siga siendo uno de los temas más relevantes en cuanto a la exclusión social de las personas con discapacidad (Anaut et al., 2017).

En lo relacionado con la educación superior y, según la información proporcionada por las 59 universidades participantes en el "Il Estudio sobre el grado de inclusión del sistema universitario español respecto de la realidad de la discapacidad" (Fundación Universia y CERMI, 2013), el número total de estudiantes con discapacidad asciende a 17.702, lo que supone un 1,3\% de la comunidad universitaria objeto de análisis. Se aprecia una ligera diferencia entre el porcentaje de alumnado con discapacidad que estudia en universidades públicas $(1,3 \%)$ y los que lo hacen en las universidades privadas $(0,9 \%)$, si bien las principales divergencias se presentan entre la tasa de universitarios con discapacidad que cursan sus estudios en universidades de modalidad presencial $(0,9 \%)$ y en universidades de modalidad a distancia $(3,1 \%)$ (Fundación Universia y CERMI, 2013).

Un tema que preocupa, especialmente a los estudiantes con discapacidad, es el relativo a la metodología de evaluación. En muchas ocasiones, la forma evaluar no está adaptada a las necesidades y características del 
alumnado con discapacidad. Ante esta realidad, cobran fuerza dos factores importantes en la consecución de la igualdad de oportunidades y no discriminación: por un lado la accesibilidad universal y el diseño para todas las personas. Por otro, la formación y experiencia de los profesionales en materia de discapacidad (Martínez Martín, 2010). La solución se fundamenta en aprovechar los recursos que, de forma natural existen en la institución universitaria, como elementos que facilitan el proceso de inclusión de todos los estudiantes en la vida universitaria.

Los factores contextuales representan el trasfondo total, tanto de la vida de un individuo como de su estilo de vida. Incluyen los factores ambientales y los factores personales que pueden tener un efecto en la persona (Organización Mundial de la Salud, 2001). Los factores ambientales constituyen el ambiente físico, social y actitudinal en el que las personas viven y desarrollan sus vidas, éstos pueden tener una influencia negativa o positiva en el desempeño ocupacional del individuo en la sociedad. Uno de los principales factores contextuales que puede afectar al desempeño de las personas con discapacidad son las actitudes.

La actitud es una predisposición aprendida para responder consistentemente de un modo favorable o desfavorable ante personas o grupos de personas, objetos sociales y situacionales. Así pues, es considerada como algo que se adquiere y, por tanto, que se puede modificar. Se ha evidenciado que las creencias y actitudes de los docentes repercuten en sus prácticas educativas, ya que pueden condicionar en gran medida el éxito en la aproximación educativa a la discapacidad (Gómez e Infante, 2004).

Las actitudes pueden suponer, para las personas con discapacidad, mayores barreras que las derivadas de su propia discapacidad, influyendo enormemente en su adaptación a los diferentes contextos, tanto académico, social, como laboral. Las actitudes de los docentes hacia la enseñanza parecen ser un factor clave para una inclusión exitosa, ya que una actitud favorable repercutirá no sólo en una mayor comprensión y ayuda a las necesidades individuales, sino al desarrollo de nuevos planteamientos didácticos que beneficiará a todo el alumnado, mejorando, en consecuencia, la calidad de la educación (Martínez Martín y Bilbao, 2011). El modo en el que el profesor responde a las necesidades de sus alumnos/as es una variable mucho más poderosa para determinar el éxito de la inclusión que cualquier estrategia administrativa o curricular. A través de la actitud positiva del profesorado se favorece que el alumno se sienta valorado en las aulas y perciba que tiene las mismas oportunidades y derechos (Martínez Martín y Bilbao, 2011).

A pesar de su importancia, no se suelen tener en cuenta las actitudes hacia la discapacidad en los currículos y proyectos docentes. Así, Verdugo et al. (1995) señalan que: "a pesar de la importancia primordial de las actitudes hacia las personas con discapacidad para lograr una inclusión real, aún son pocos los centros e instituciones que incluyen, como parte importante de su quehacer profesional, actividades, objetivos, y contenidos dirigidos a evaluar y mejorar las actitudes" (Moreno et al., 2006).

Desde hace varios años, el interés desde diferentes ámbitos por atender las necesidades del colectivo de personas con discapacidad, al igual que la proliferación de investigaciones dedicadas a su inclusión, se ha incrementado notablemente (Suriá et al., 2011). La evidencia científica señala que las actitudes son un aspecto fundamental en el éxito de las personas con discapacidad en sus procesos de inclusión y desarrollo de los procesos de aprendizaje académico (Suriá, 2011).

Diversos estudios indican que los jóvenes universitarios muestran una actitud positiva hacia los compañeros con discapacidad, sin embargo, es preciso atender en mayor medida y conocer las variables determinantes 
de las conductas inclusivas (Novo-Corti et al., 2011). Suriá (2011) ha destacado la existencia de un mayor grado de sensibilización hacia la discapacidad por parte de aquellos estudiantes que tenían relación con compañeros con discapacidad (Novo-Corti et al., 2011). En el estudio de Gómez e Infante (2004) los resultados concluyen que todos los estudiantes universitarios muestran actitudes positivas hacia la educación multicultural, señalando que variables como la edad o el contacto no son factores explicativos de su actitud. Sin embargo detectó, como Gughwan (2001), una tendencia a mejorar las actitudes conforme se realizan los estudios en los últimos años de carrera (Novo-Corti et al., 2011).

En relación con el tipo de discapacidad, en el estudio de Alarcón y Busqués (2014) una cuarta parte de los estudiantes muestra reticencia a desarrollar un trabajo con un compañero con discapacidad auditiva o visual, mostrando actitudes más positivas ante el hecho de trabajar con estudiantes con discapacidad física. En este mismo estudio se denotan resultados positivos en la percepción de la necesidad de realizar adaptaciones para estudiantes con discapacidad, opiniones asociadas a la interacción con compañeros con discapacidad y disposición a apoyar el proceso de inclusión de éstos en la comunidad universitaria (Alarcón y Busqués, 2014).

Por otro lado, Gómez e Infante (2004) y Alonso et al. (2007) hallaron en sus resultados, diferencias significativas en función de la rama de conocimiento de los estudiantes universitarios, de manera que las actitudes son más positivas en los estudiantes de la rama de Humanidades y Educación que en el resto de áreas de conocimiento (Novo-Corti et al., 2011).

Lissi et al. (2014) enfatizan la importancia de la formación en temas relacionados con la discapacidad, indicando en su estudio que las tutorías pueden ser un importante recurso para apoyar a estudiantes con discapacidad en contextos universitarios en el ámbito académico y social y tienen un efecto preventivo en la deserción universitaria. En las tutorías implementadas en dicho estudio, se ha detectado que los participantes aprendieron a reconocer los apoyos necesarios para eliminar las barreras en la participación y el aprendizaje, destacándose las adaptaciones curriculares y el uso de herramientas tecnológicas que favorezcan la inclusión. Sin embargo, en el estudio de Rodríguez-Martín y Álvarez-Arregui (2014), se ha detectado una percepción negativa de los estudiantes hacia la adaptación de los objetivos, contenidos y evaluación en el ámbito académico hacia los estudiantes con discapacidad.

Trabajos como el de Higbee et al. (2007) demuestran que la discapacidad en la universidad es reconocida por los estudiantes como una experiencia educativa enriquecedora, tal y como los autores Lissi et al. (2013) y Salinas et al. (2013) confirman la importancia que tienen los compañeros sin discapacidad en el proceso de inclusión universitaria, consignándolo como un valor significativo en su quehacer universitario, demostrado a través de las relaciones interpersonales fundadas en la colaboración y en la amistad. Sin embargo, como recuerdan Borland y James (1999), Reindal (1995) o Tinklin y Hall (1999) el ambiente universitario puede ser en algunos casos discapacitante (Moriña et al., 2013). Castellana y Bars (2006) han concluido también que el aula no dispone de los recursos precisos para favorecer la inclusión, y que el profesorado no imparte las clases utilizando las metodologías adecuadas para que puedan participar plenamente en ellas (Moriña et al., 2013).

En el estudio de Moneo y Anaut (2017), un $25 \%$ de los estudiantes participantes confirman que mantienen ideas y actitudes poco inclusivas hacia sus pares con discapacidad pero, a su vez, reconoce el déficit 
formativo, de visibilización e información de la realidad de la discapacidad en el entorno universitario. En consecuencia, aún es preciso conocer diversos aspectos que determinan el tipo de actitudes de la comunidad universitaria hacia los estudiantes con discapacidad con el fin de favorecer la existencia de actitudes favorables y alcanzar una comunidad universitaria inclusiva.

Por todo ello, se ha determinado que el objetivo general de la presente investigación es determinar las actitudes de los estudiantes y profesores hacia la discapacidad y conocer las variables que modifican dichas actitudes; que se ha concretado en otros objetivos específicos:

- Establecer la percepción que los docentes, con relación previa con la discapacidad, tienen sobre las adaptaciones.

- Conocer la opinión de docentes y estudiantes sobre los deberes del profesorado para con los estudiantes con discapacidad y los conocimientos que tienen sobre sus necesidades.

- Estudiar la percepción sobre la accesibilidad y el grado de sensibilización de la comunidad universitaria.

\section{Método}

Hemos llevado a cabo un estudio descriptivo de prevalencia con el que se pretende describir el fenómeno de estudio especificando sus características y los perfiles de los participantes. El estudio se ha desarrollado en la Universidade da Coruña (UDC), perteneciente al Sistema Universitario de Galicia (SUG).

\section{- Participantes}

La muestra estuvo compuesta por docentes y estudiantes de la UDC. Se ha accedido a la muestra a través del Servicio de Atención a la Diversidad (ADI) de la propia universidad. La muestra analizada constaba de 121 docentes y 345 estudiantes, tamaño muestral suficiente para estimar los parámetros de interés con una precisión del $8,5 \%$ y $5,2 \%$ respectivamente. El $63 \%$ de los participantes fueron mujeres. El $80 \%$ poseía algún tipo de relación con la discapacidad, de éstos un $19 \%$ indica que se trata de una relación de amistad. El $54,5 \%$ de los docentes y el 30,5\% de los estudiantes fueron hombres. La media de edad de los docentes fue de 49,9 años ( $D T=10.5)$, y la de los estudiantes se situó en 24,2 años ( $D T=6.3)$. En cuanto a la experiencia laboral de los docentes, la media fue de 16 años $(D T=10.1)$, y la media de años de estudios cursados por los estudiantes fue de 4,4 años $(D T=3.1)$.

\section{- Instrumentos}

La variable independiente, las actitudes, ha sido evaluada a través de la herramienta "Cuestiones sobre Universidad y Discapacidad" (Rodríguez-Martín y Álvarez-Arregui, 2013) (CUNIDIS), basada en una escala tipo Likert de 5 puntos. Está compuesta por 40 ítems y estructurada en cuatro bloques de contenido: los deberes del profesorado, las acciones que el profesorado realiza, la accesibilidad y la sensibilización de la comunidad universitaria. 
Se han realizado también mediciones relacionadas con las características de los participantes: sociodemográficas (edad, sexo y estado civil), académicas (estudios cursados o en curso, facultad donde trabaja/ estudia y años de experiencia/cursados) y discapacidad (tener discapacidad, relación con la discapacidad y tipología de relación con la discapacidad).

\section{- Procedimiento}

El procedimiento de recogida de datos ha sido a través de una encuesta online, autoadministrada y anonimizada.

\section{- Análisis estadístico}

En primer lugar se ha comprobado la fiabilidad de las subescalas del cuestionario CUNIDIS. A continuación se ha realizado un estudio descriptivo de las variables incluidas en el estudio. Las relaciones entre las variables se han analizado mediante un ANOVA. La muestra de profesores y la de estudiantes se ha considerado de manera independiente.

\section{Resultados}

\section{- Análisis de fiabilidad}

Se ha llevado a cabo un análisis de fiabilidad de la escala CUNIDIS para determinar si la fiabilidad obtenida por los autores se mantenía en nuestra muestra. Los resultados indicaron unos índices de fiabilidad aceptables en los bloques de deberes del profesorado $(\mathrm{a}=.86)$; acciones que llevan a cabo por el profesorado $(\mathrm{a}=.93)$; accesibilidad $(\mathrm{a}=.85)$ y, en menor medida, en el bloque de sensibilización de la comunidad universitaria $(\mathrm{a}=.79)$.

\section{- Resultados descriptivos}

En lo que respecta a los datos sociodemográficos de los participantes, el $65,8 \%$ de los docentes estaban casados, un $5 \%$ divorciados y un $28,8 \%$ solteros. En el caso de los estudiantes, la inmensa mayoría (96\%) estaban solteros. Un 3,3\% de los docentes y un $4,2 \%$ de los estudiantes experimentaba algún tipo discapacidad. En cuanto a su relación con la discapacidad, un $86,7 \%$ de los docentes y un $73 \%$ de los estudiantes había tenido relación previa con alguna persona con discapacidad. La mayor parte de docentes y estudiantes en el estudio provenían de la rama de ciencias sociales y jurídicas, como se observa en la tabla 1.

Como se observa en la tabla 2, la mayor parte de los docentes tiene contacto con la discapacidad a través de sus alumnos/as, mientras que en el caso de los estudiantes este contacto es más heterogéneo. 


\begin{tabular}{|c|c|c|}
\hline & Docentes & Estudiantes \\
\hline Artes y Humanidades & 6,2 & 6 \\
\hline Ciencias & 8,6 & 6,5 \\
\hline Ciencias de la Salud & 9,5 & 14,3 \\
\hline Ciencias Sociales y Jurídicas & 48,3 & 42,6 \\
\hline Ingeniería y Arquitectura & 28,4 & 30,7 \\
\hline
\end{tabular}

Fuente: elaboración propia.

\begin{tabular}{l}
\hline Tabla 2. Tipo de contacto con la discapacidad (en \%) \\
\cline { 2 - 3 } \multicolumn{1}{l|}{} \\
\begin{tabular}{|l|c|c|}
\hline Alumnos con discapacidad & Docentes & Estudiantes \\
\hline Amistad & 47,6 & 14,5 \\
\hline Compañero/ de trabajo & 19,4 & 29 \\
\hline Familiar & 21,4 & 1,6 \\
\hline Pareja & - & 1,2 \\
\hline Otra & 10,7 & 27 \\
\hline
\end{tabular}
\end{tabular}

Fuente: elaboración propia.

En cuanto al colectivo de participantes con discapacidad en nuestro estudio, 18 en total, el $50 \%$ pertenece a cada sexo. Por otro lado, el $77 \%$ indicó que su estado civil es soltero/a y el $41 \%$ que pertenece a la rama de Ciencias Sociales y Jurídicas. En cuanto a su vinculación con la discapacidad, el 83 \% mantiene contacto con otras personas con discapacidad, en el $50 \%$ de los casos debido a relaciones de amistad.

A continuación se ofrecen los datos descriptivos más relevantes según los bloques evaluados por la escala CUNIDIS:

Deberes del profesorado para con los estudiantes con discapacidad. En este bloque de contenido se analizan las percepciones sobre los deberes del profesorado para con los estudiantes con discapacidad $y$, entre los resultados más relevantes, se ha detectado que mientras que el $87 \%$ de la muestra de los docentes está totalmente o muy de acuerdo con adaptar los materiales utilizados por las personas con discapacidad en las actividades del aula; el $41 \%$ del grupo de profesores está totalmente en desacuerdo o poco de acuerdo con que se deban adaptar los criterios de evaluación y cualificación para las personas con discapacidad. Sin embargo, los estudiantes muestran porcentajes inferiores en las respuestas a los ítems anteriores dados por los docentes, $76 \%$ y $31 \%$, respectivamente.

La totalidad de los participantes se muestra totalmente de acuerdo en adaptar los materiales utilizados en las actividades docentes (53\%), los criterios de evaluación (25\%), y las actividades que se desarrollan en 
las diferentes materias (45\%). Por otro lado, docentes y estudiantes indican estar totalmente en desacuerdo o poco de acuerdo con adaptar el tiempo para la realización de exámenes o entregas de trabajos (27\%), adaptar los objetivos de las materias (39\%) o los contenidos de las asignaturas (39\%).

Actuaciones de los docentes en el aula hacia los estudiantes con discapacidad. La cuarta parte de los encuestados está totalmente en desacuerdo con que en la actualidad en la realidad del aula se realiza la adecuada adaptación de contenidos de las materias que se imparten a los estudiantes con discapacidad. En concreto, más de la mitad de los docentes que afirman haber tenido contacto con la discapacidad indican que no adaptan los contenidos de las materias para las personas de este colectivo, dato que queda reflejado en que están totalmente en desacuerdo o poco de acuerdo (57\%) con que en la realidad del aula se apliquen este tipo de adaptaciones.

Participación de los estudiantes con discapacidad en la comunidad universitaria. Menos de la mitad de los participantes (43\%) están muy o totalmente de acuerdo con que las personas con discapacidad participan en todas las actividades que se proponen en el aula. El $57 \%$ de los encuestados consideran que la facultad no presenta barreras arquitectónicas.

Sólo uno de cada cuatro docentes (26\%) manifiesta que está muy o totalmente de acuerdo con que los materiales impresos o audiovisuales que se entregan en las clases están adaptados para personas con discapacidad. Por otro lado, la mitad de los profesores (49\%) considera insuficientes los apoyos humanos y materiales para seguir las clases. En cuanto a los estudiantes, estos muestran porcentajes ligeramente inferiores en las respuestas referentes a la accesibilidad de los materiales (17\%), mientras que presentan porcentajes similares (48\%) en cuanto a los apoyos.

Sensibilización de la comunidad universitaria hacia los estudiantes con discapacidad. Tres de cada cuatro encuestados (75\%) creen firmemente que un estudiante con discapacidad puede ser un buen profesional el día de mañana. Específicamente en el grupo de profesores la cifra se eleva al $78 \%$. El $65 \%$ de los docentes indica que está totalmente en desacuerdo o poco de acuerdo con que el profesorado de la universidad esté formado en las competencias adecuadas para dar respuesta a las necesidades de los estudiantes con discapacidad.

Diferencias en las actitudes hacia la discapacidad. En primer lugar, se observaron diferencias entre profesores y estudiantes en las actitudes hacia las posibilidades de participación $(t(446)=4.91, p<.001)$, los profesores tienen una mejor percepción de las posibilidades de participación del alumnado con discapacidad $(M=3.12, D T=.76)$ que los estudiantes $(M=2.72, D T=.76)$. Los profesores también perciben una mayor sensibilización que el alumnado $(t(439)=3.00 p<.01 ; M=3.44, D T=.62$ vs. $M=3.24, D T=.64)$.

Mientras que en la muestra de profesores no se encontraron diferencias significativas en cuanto al sexo, edad, años de experiencia, contacto con la discapacidad o el hecho de experimentar una discapacidad, se observaron una serie de diferencias en el caso de los estudiantes.

Se encontró una diferencia entre los estudiantes hombres y mujeres en lo que respecta a la percepción de los deberes del profesorado hacia los estudiantes con discapacidad $(t(334)=2.10, p<.05)$; las mujeres tienen una percepción de que es más necesario adaptar las actividades, materiales y evaluaciones $(M=3.77$, 
$D T=.84)$ que sus compañeros varones $(M=3.56, D T=.86)$. Las mujeres también tienen una peor percepción hacia el grado de accesibilidad de la comunidad universitaria $(t(327)=2.67 p<.05$; mujeres $M=2.64, D T=.73$; hombres $M=2.88, D T=.80)$.

Con respecto a la edad de los participantes, los estudiantes muestran una tendencia a que, a mayor edad, peor percepción de grado de sensibilización en el ámbito universitario ( $r=-.12, p<.05)$. En cuanto a los años de estudios cursados, a más años académicos de experiencia perciben un peor grado tanto de accesibilidad $(r=-.14, p<.05)$ como de sensibilización $(r=-.17, p<.01)$ de la comunidad universitaria.

También se observó entre los estudiantes con discapacidad una actitud más positiva hacia las posibilidades de participación en el ámbito universitario $(t(325)=2.06, p<.05 ; M=3.13, D T=.93)$, en comparación con sus compañeros sin discapacidad $(M=2.70, D T=.75)$. Sin embargo, no se observaron diferencias significativas por el hecho de tener o no contacto con personas con discapacidad.

Centrándonos en la percepción de los deberes hacia los estudiantes con discapacidad, a través de un ANOVA de un factor, se observaron una serie de diferencias significativas en función de la rama de estudios de los participantes $(F(4,327)=7.13, p<.001)$. Los contrastes post-hoc utilizando el nivel de confianza de Bonferroni muestran diferencias significativas de los estudiantes de Ingeniería y Arquitectura con los de Artes y Humanidades $(p=.06)$, Ciencias de la Salud $(p<.001)$, aunque no con los estudiantes de Ciencias. Los estudiantes de Ingeniería y Arquitectura puntuaron significativamente menos en percepción de derechos $(M=3.38, D T=.79)$, que los de Arte y Humanidades $(M=3.94, D T=.72)$, Ciencias de la Salud $(M=3.99, D T=.71)$, Ciencias Sociales y Jurídicas $(M=3.85, D T=.86)$ y aunque no de manera significativa, también menos que los de Ciencias $(M=3.57, D T=.99)$.

\section{- $\quad$ Análisis de varianza}

Se llevó a cabo un análisis de varianza para determinar qué variables explican mejor las diferencias en la percepción de los derechos de los estudiantes con discapacidad entre los estudiantes; en el caso de los profesores no se encontraron diferencias significativas y tampoco con respecto a otras subescalas del cuestionario CUNIDIS. El modelo de ANOVA elaborado incluye como predictores el sexo de los participantes, su contacto con personas con discapacidad y la rama de conocimiento de los estudiantes. El resto de variables consideradas previamente no fueron incorporadas al modelo final al no ofrecer ninguna mejora sustancial. El modelo elaborado fue significativo $(F(18,313)=2.60, \mathrm{p}<.001)$ y con un tamaño del efecto moderado ( $\eta^{2}$ parcial=.13). El modelo indicó un efecto principal del conocimiento de personas con discapacidad $\left(F(1,330)=4.20, p<.05 ; \eta^{2}\right.$ parcial $\left.=.01\right)$ y de la rama de conocimiento donde se cursan estudios $\left(F(4,327)=7.27, \mathrm{p}<.001 ; \eta^{2}\right.$ parcial=.08). No hubo un efecto principal del sexo pero sí una interacción entre sexo y conocimiento de personas con discapacidad $\left(F(1,330)=5.02, p<.05 ; \eta^{2}\right.$ parcial=.02). Por último, se observó también una interacción entre el contacto con personas con discapacidad y la rama de conocimiento $\left(F(4,327)=2.83, \mathrm{p}<.05 ; \eta^{2}\right.$ parcial $\left.=.02\right)$.

Las medias marginales estimadas indicaron una actitud más favorable a adaptar los recursos en la universidad entre los participantes que no tenían relación con personas con discapacidad ( $M$ marginal estima$d a=3.89$ vs. $M$ marginal estimada=3.89) y medias estimadas inferiores en los estudiantes de Ingeniería y arquitectura ( $M$ marginal estimada=3.33) y Ciencias ( $M$ marginal estimada=3.19) con respecto a los de Artes 
y Humanidades ( $M$ marginal estimada=3.99); Ciencias sociales y Jurídicas ( $M$ marginal estimada $=3.85$ ) y Ciencias de la salud ( $M$ marginal estimada $=4.32$ ).

Con respecto a la interacción entre sexo y el contacto con personas con discapacidad, se observó que mientras que en el caso de las mujeres las actitudes no se alteraban en función del contacto, en el caso de los hombres las actitudes favorables aumentaban cuando no se tenía un contacto directo de personas con discapacidad, como puede verse en el gráfico 1:

Gráfico 1: Interacción entre sexo y conocimiento de personas con discapacidad

\begin{tabular}{r}
4.2 \\
\hline 4.0 \\
\hline \\
\hline .8 \\
\hline 3.6 \\
\hline \\
\hline 3.4 \\
\hline 3.2 \\
\hline 3.0 \\
\hline \\
Hombre \\
Muier
\end{tabular}

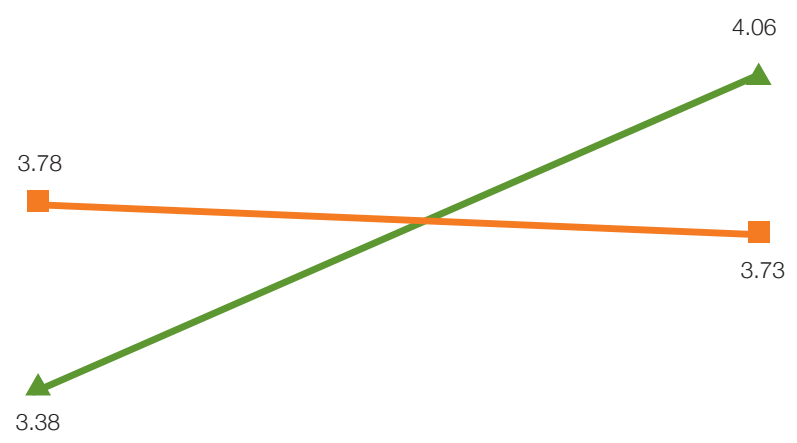

Fuente: elaboración propia.

En cuanto a la interacción entre rama de conocimiento y conocimiento de la discapacidad se encontró que en algunas ramas, como Arte y Humanidades e Ingeniería y Arquitectura el desconocimiento de personas con discapacidad va asociado a una menor percepción de la necesidad de adaptar los recursos, mientras que en Ciencias y Ciencias de la Salud, este conocimiento va asociado a una mayor percepción de necesidad. 
Gráfico 2: Interacción entre rama de estudios y conocimiento de personas con discapacidad

$\begin{array}{r}5.0 \\ \hline 4.5 \\ \hline 4.0 \\ \hline 3.5 \\ \hline \\ \hline 3.0 \\ \hline 2.5 \\ \hline\end{array}$

Arte y Humanidades

Ciencias

Ciencias de la Salud

Ciencias Sociales y Jurídicas

Inqeniería v Arquitectura

Fuente: elaboración propia.

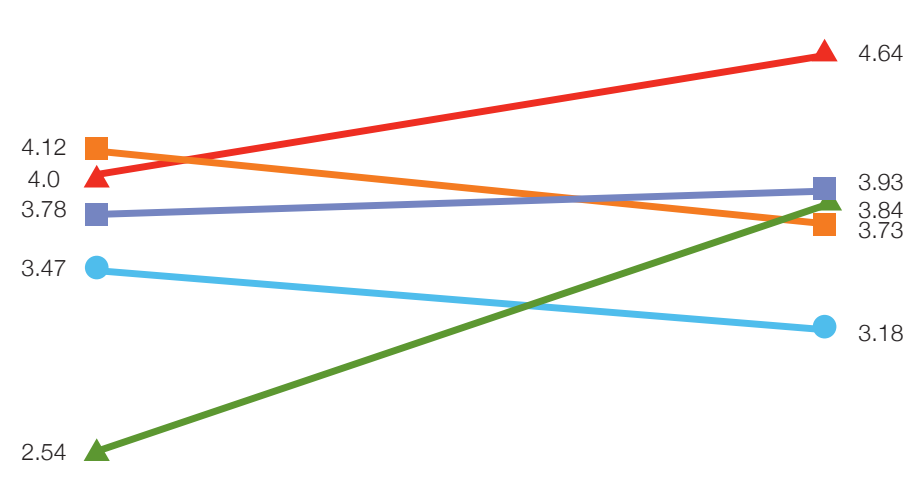

Sí

No

\section{Discusión}

Los resultados obtenidos en la presente investigación pretenden ofrecer una perspectiva general sobre cómo son las actitudes de los docentes y estudiantes de la Universidad de A Coruña, hacia los estudiantes con discapacidad. Estas actitudes vienen determinadas, en este caso, por los diferentes bloques de contenido de la encuesta CUNIDIS: adaptación de las materias, la realidad del aula, la accesibilidad y, por último, la sensibilización.

El $80 \%$ de los participantes señalaron que tienen algún tipo de relación con personas con discapacidad. Por lo que se puede indicar que esta experiencia es algo muy común en el ámbito en el que se desarrolla la investigación, ya no sólo en el educativo, sino en la vida cotidiana de los encuestados.

Los resultados de nuestro estudio son concordantes con los de Rodríguez-Martín y Álvarez-Arregui (2015), con respecto a la vinculación con la discapacidad, en el que el $77 \%$ de los docentes y el $74 \%$ de los estudiantes manifestaron tener contacto con este colectivo, lo que refuerza que la relación de docentes y estudiantes de nuestro estudio con personas con discapacidad no es un hecho puntual. 
Por otra parte, en el estudio de Fiuza (2012), llevado a cabo en una facultad de Lugo, identificaron que la relación de los participantes con las personas con discapacidad viene dada mayoritariamente a través del contacto con algún familiar. Por último, en cuanto a la relación con la discapacidad, de la totalidad de estudiantes participantes en el estudio de Novo-Corti et al. (2011), sólo el 36 \% indicaron tener relación con este colectivo.

A fin de tratar de averiguar la presencia e influencia de ciertos factores en el forjamiento de actitudes hacia el mencionado colectivo se han realizado análisis de correlación entre las variables recogidas.

Actitudes hacia la adaptación de las materias. En conjunto, la mitad de los participantes, indica que es necesario adaptar los materiales empleados en las actividades docentes, mientras que sólo la cuarta parte señala que es preciso adaptar los criterios de evaluación y de cualificación para el alumnado con discapacidad, en base a sus necesidades.

Estos resultados no son del todo coincidentes con los datos publicados por Rodríguez-Martín y ÁlvarezArregui (2015), ya que si bien en su trabajo, como en el nuestro, los estudiantes presentan respuestas favorables hacia la adaptación de las actividades, de los materiales utilizados y de las prácticas, la mayoría de los docentes, por el contrario, consideraban que no deben adaptar los objetivos, los contenidos, los instrumentos ni los criterios de evaluación. Esta diferencia, pensamos, puede deberse a que la adaptación de estos criterios a favor de los estudiantes con discapacidad puede entenderse por el profesorado universitario como un trato de favor hacia este colectivo. En este sentido coincide con las reflexiones del estudio publicado por Moriña et al. (2013) en el que, además, indican que la actitud de los docentes no siempre es todo lo adecuada que debería ante las adaptaciones.

La actitud favorable del grupo de alumnos/as hacia la adaptación de diversas acciones docentes para las personas con discapacidad no es nada nuevo. Ya en el año 2011, en el trabajo publicado por Novo-Corti et al. (2011) los estudiantes manifestaron que asumen la necesidad de políticas inclusivas, por lo que las actuaciones y adaptaciones bajo el apoyo institucional serían aceptadas con agrado.

Actitudes hacia la acción docente que realiza el profesorado. Con respecto a las acciones llevadas a cabo en la realidad del aula, el $25 \%$ de los participantes indicaron que en la actualidad no se realizan las adaptaciones hacia los estudiantes con discapacidad correspondientes a los contenidos de las materias que se imparten.

Más de la mitad de los docentes lo ha corroborado. Siguiendo la misma línea que en nuestro estudio, los docentes encuestados por Rodríguez-Martín y Álvarez-Arregui (2015) también mencionaron que en la práctica real del aula no se adaptan los objetivos ( $80 \%)$, los contenidos $(80 \%)$ ni los instrumentos y criterios de evaluación (67\%), mientras que los estudiantes coinciden con la tendencia del profesorado, identificando que no se realiza la adaptación de los objetivos $(36,8 \%)$ ni de los contenidos $(40 \%)$ de las diferentes materias. Realizar las adaptaciones necesarias para cada estudiante que lo necesite es un medio a través del cual garantizar la igualdad de oportunidades y la inclusión en el acceso y desarrollo de sus estudios superiores, ahí radica su importancia.

Por tanto, los resultados obtenidos reflejan que la mayoría de los encuestados creen que es necesario realizar adaptaciones para los estudiantes con discapacidad, pero a su vez, reconocen que en la actualidad 
no se están implementando realmente, a pesar de ser necesarias para que estos estudiantes desarrollen su actividad académica de manera satisfactoria.

En el estudio de Moriña et al. (2013), los participantes afirmaron que el profesorado no se muestra predispuesto a facilitar, por adelantado, el material docente que se va a utilizar para la docencia. En la misma línea también han identificado la falta de previsión de los docentes para la adaptación de los recursos y materiales con el tiempo necesario.

Por otra parte, y considerando que el $57 \%$ de los docentes con contacto previo con la discapacidad no realiza dichas adaptaciones, se puede inferir que el simple hecho de mantener algún tipo de contacto con personas con discapacidad, no predispone a los docentes a realizar las adaptaciones correspondientes. Esto puede ser causado por el desconocimiento hacia la manera de elaborar las antedichas adaptaciones, ya que actualmente no existe formación de carácter obligatorio en la que el profesorado adquiera las habilidades para llevar a cabo adaptaciones de currículo. Los resultados del estudio de Rodríguez-Martín y Álvarez-Arregui (2015) apoyan los de nuestra investigación, ya que el profesorado y los estudiantes consideran que en la práctica real del aula no se adaptan los contenidos de las diferentes materias impartidas.

Participación de los estudiantes con discapacidad en la comunidad universitaria. Menos de la mitad de los participantes consideran que los estudiantes con discapacidad participan en todas las actividades que se proponen de manera cotidiana en el aula. Esto puede indicar que la inclusión del colectivo no es totalmente completa, ya que no todos los estudiantes participan de la misma manera durante la docencia. Este hecho puede estar influenciado por la existencia de diversas barreras arquitectónicas, de la comunicación, actitudinales, sociales o culturales.

Por otro lado, el 50,8\% ha considerado que los materiales impresos o audiovisuales que se entregan para llevar a cabo la docencia están adaptados para las personas con discapacidad. De nuevo, esto parece indicar que es preciso garantizar una formación especializada en accesibilidad educativa dirigida a los docentes, que permita realizar las adaptaciones específicas para que los materiales utilizados por éstos sean accesibles para todos los estudiantes. Este dato es apoyado por los resultados obtenidos en la investigación de Rodríguez-Martín y Álvarez-Arregui (2015) en la que tanto profesorado (49\%) como estudiantes (57\%) reconocen que se adaptan algunos materiales y se utilizan metodologías de apoyo para el seguimiento de las clases por parte del alumnado con discapacidad.

En cuanto a los apoyos humanos y materiales, en nuestro estudio se ha evidenciado un dato que sigue la línea de los presentados anteriormente, ya que la mitad de los participantes (49\%) afirmó que éstos no cuentan con las ayudas necesarias para poder seguir de manera satisfactoria el desarrollo de las clases. Es preciso señalar que aunque se utilicen tecnologías de apoyo y se adapten ciertos materiales, esto no es suficiente ya que, en muchas ocasiones, su realización no se lleva a cabo de una manera correcta. Por este motivo, puede ser que se llegue a la conclusión de que ni los apoyos humanos ni los materiales son suficientes para garantizar la participación de los estudiantes con discapacidad en las aulas, sino que se debería potenciar el correcto asesoramiento para el buen uso de las tecnologías de apoyo.

Sensibilización de la comunidad universitaria. En los resultados se ha evidenciado que el $75 \%$ de los participantes de este estudio considera que un estudiante con discapacidad puede ser un buen profesional 
y desarrollar su labor de manera satisfactoria. Esto parece indicar una actitud positiva hacia la inclusión educativa, y posteriormente laboral, de los estudiantes con discapacidad, ya que se han detectado datos similares en el estudio de Rodríguez-Martín y Álvarez-Arregui (2015). Por tanto, puede observarse que, a pesar de la multitud de creencias y prejuicios que puedan existir ante las personas con discapacidad, actualmente estas actitudes están cambiando. Se evidencia la realidad y por tanto, la existencia de una actitud positiva hacia que una persona, a pesar de la discapacidad que tenga, puede desarrollar su actividad estudiantil y laboral de igual manera que una persona que no presente dicha condición.

En los estudios de Rodríguez-Martín y Álvarez-Arregui (2015) y de Abu-Hamour (2013) se corroboran los resultados de este estudio, en los que los participantes, docentes y estudiantes, consideran que el profesorado no está formado para dar respuesta adecuada a los estudiantes con discapacidad, hecho que puede ser debido a que no ha recibido una formación específica y especializada.

Como punto en común con nuestro estudio, Moriña et al. (2013) también identificaron que el profesorado carece de la formación suficiente para atender las necesidades de los estudiantes con discapacidad, hecho que puede dificultar la progresión académica de éstos. Según se ha argumentado en dicho estudio, no ha existido la suficiente preparación del profesorado previamente. Este hecho se ha convertido en una de las principales barreras en el desarrollo académico y no se trata siempre de una inadecuada actitud por parte de los docentes, sino de una ausencia de formación sobre la atención a la discapacidad. Igualmente CasteIlana y Sala (2005), Moswela y Mukhopadhyay (2011) y Sánchez Palomino (2009) hallaron que existe un gran desconocimiento hacia la discapacidad y que el profesorado debería adquirir unas competencias mínimas para darle una respuesta de calidad.

Diferencias en las actitudes hacia la discapacidad. Se ha detectado una asociación entre los años de estudios cursados por los estudiantes y la relación con la discapacidad; en cuanto a este factor, en la actualidad existen diferencias relevantes entre los resultados obtenidos en diferentes estudios. Por ejemplo, Suriá (2011), en su trabajo en la Universidad de Alicante, ha detectado diferencias estadísticamente significativas en las actitudes de los estudiantes en base al curso en el que desarrollan sus estudios. Sin embargo, existen discrepancias entre si la mayor o menor experiencia en los cursos repercute en unas mejores o peores relaciones, puesto que Abu-Hamour (2013) en su estudio, llevado a cabo en Jordania, detectó que los miembros de la facultad con menos años de experiencia presentan actitudes más positivas hacia los estudiantes con discapacidad. Por otra parte, Alonso et al. (2007) han determinado que en cuanto al nivel de estudios cursado existen diferencias significativas, presentando actitudes más positivas los estudiantes de último curso.

En nuestro estudio se ha podido determinar que la rama de conocimiento tiene influencia en el tipo de relaciones con la discapacidad, tal y como en el estudio de Rodríguez-Martín y Álvarez-Arregui (2015), en el que se ha detectado que el profesorado y los estudiantes de la rama de Ciencias Sociales y Jurídicas, y en menor medida los adscritos a Ciencias de la Salud, tienen puntuaciones superiores y más favorables hacia las cuestiones presentadas con respecto a los de la rama de Arquitectura e Ingeniería. Esto puede ser debido a los contenidos que se desarrollan en las antedichas ramas de conocimiento, ya que en las ramas Sociales, Jurídicas y en las de Ciencias de la Salud, el conocimiento de la discapacidad es un contenido a tratar en diversas materias, en mayor medida que en las ramas de Arquitectura o en las Ingenierías, a pesar de la importancia con la que cuenta el conocimiento de la discapacidad en el ámbito de la Arquitectura, ya que ayudaría a garantizar la accesibilidad universal. 
Las diferencias entre estas áreas es indiscutible, primero por los perfiles profesionales del profesorado y, segundo, por las características de las titulaciones que se incluyen en estas áreas, algunas de ellas vinculadas de forma directa con el mundo de la discapacidad. Además de estas divergencias, el contacto con personas con discapacidad constituye la variable que condiciona el nivel y calidad de las respuestas en la línea de estudios previos. Alonso et al. (2007) han obtenido que los estudiantes del área de Humanidades presentan puntuaciones más positivas, sin embargo, el área de Educación muestra puntuaciones más bajas. Polo et al. (2011), que han realizado una investigación en una universidad de Colombia teniendo como participantes a los estudiantes de Ciencias Sociales y Psicología, en sus resultados también evidenciaron que el alumnado presentaba actitudes positivas hacia las personas con discapacidad, existiendo diferencias según la titulación cursada y siendo más relevante el hecho de mantener contacto previo con personas con discapacidad.

El desconocimiento de la discapacidad, las actitudes hacia este colectivo y sus repercusiones en el desarroIlo, tanto personal como académico, del alumnado con discapacidad son un factor clave en el cual es preciso intervenir. Es necesario implantar programas de sensibilización que potencien un mayor acercamiento a la temática de la discapacidad, favoreciendo a su vez diversos aspectos de gran importancia como son el derecho a una educación de calidad, en igualdad de oportunidades e inclusiva.

\section{- Limitaciones del estudio}

Entre las limitaciones del estudio se considera relevante que no se ha utilizado una metodología mixta con la que se podrían añadir datos de tipo cualitativo que enriquecerían los resultados del estudio.

\section{Conclusiones}

En base a los resultados obtenidos en el presente estudio, se puede concluir que los participantes son conscientes de las carencias existentes aún hoy día en relación al grado de sensibilización y de accesibilidad de la comunidad universitaria. Hecho que refleja que la inclusión educativa en la educación superior sigue siendo un reto para las comunidades universitarias.

La educación es un pilar fundamental para la inclusión real de las personas con discapacidad por ello, debe ser la base de todas las administraciones públicas asegurar la existencia de un sistema educativo lo más inclusivo posible, con el objetivo de alcanzar la igualdad de oportunidades en el desarrollo de las actividades formativas y laborales de las personas con discapacidad. 


\section{Referencias bibliográficas}

Abu-Hamour, B. (2013): "Faculty Attitudes toward Students with Disabilities in a Public University in Jordan". International Education Studies, 6 (12): 74-81.

Alarcón, M. S. y Busqués, S. S. (2014): Actitudes de estudiantes sin discapacidad hacia la inclusión de estudiantes con discapacidad en la educación superior. Tesis doctoral. Barcelona: Universitat Autònoma de Barcelona.

Alonso, M. J. et al. (2007): "Actitudes hacia la diversidad en estudiantes universitarios". Fòrum de Recerca, 13: 476-491.

Anaut, S. et al. (2017): "La exclusión social, una problemática estructural entre las personas con discapacidad". Áreas. Revista Internacional de Ciencias Sociales, 36: 167-181.

Borland, J. y James, S. (1999): "The learning experience of students with disabilities in higher education. A case study of a UK university”. Disability \& Society, 14 (1): 85-101.

Cambois, E. (2001): Disability and social participation in Europe. Luxembourg: Office for Official Publications of the European Communities.

Castellana, M. y Bars, I. S. (2006): "La inclusión de los estudiantes con discapacidad en la universidad: un reto para la universidad española en el nuevo espacio europeo de la educación superior”. Aloma: revista de psicologia, ciències de l'educació i de l'esport Blanquerna, 18: 209-227.

Castellana, M. y Sala, I. (2005): "Estudiantes con discapacidad en aulas universitarias", en / Congreso Nacional de Universidad y Discapacidad, Universidad de Salamanca, Salamanca.

De Luna, M. (2004): "Discapacidad y aspectos sociales: la igualdad de oportunidades, la no discriminación y la accesibilidad universal como ejes de una nueva política a favor de las personas con discapacidad y sus familias. Algunas consideraciones en materia de protección social”. Revista del Ministerio de Trabajo e Inmigración, 50: 21-46.

Fiuza, M. J. (2012): "Escuela Inclusiva. Conocimientos y creencias del alumno del Grado de Maestro en Educación Infantil”. Innovación educativa, 22: 39-55.

Fundación Universia y CERMI (2013): I/ Estudio sobre el grado de inclusión del sistema universitario español respecto de la realidad de la discapacidad. Madrid: Fundación Universia y CERMI.

Gómez, V. e Infante, M. (2004): "Actitudes de los estudiantes de educación hacia la integración de personas con discapacidad y hacia la educación multicultural”. Cultura y educación, 16 (4): 371-383.

Gughwan, C. (2001): "Korean students' differential attitudes toward people with disabilities: an acculturation perspective". International Journal of Rehabilitation Research, 24 (1): 79-81.

Higbee, J. L. et al. (2007): "Student perceptions of their multicultural learning environment: A closer look". Diversity and the postsecondary experience, 3-24.

Lissi, M. R. et al. (2014): "Aprender a través de enseñar: análisis de la experiencia de tutores de estudiantes con discapacidad sensorial o motora, en un contexto universitario". Revista Latinoamericana de Educación Inclusiva, 8 (1), 109-126. 
Lissi, M. R. et al. (2013): En el camino hacia la educación superior inclusiva en Chile: fundamentos y adecuaciones curriculares para estudiantes con discapacidad sensorial y motora. Santiago de Chile: Pontificia Universidad Católica de Chile.

Martínez Martín, M. A. y Bilbao, M. C. (2011): "Los docentes de la Universidad de Burgos y su actitud hacia las personas con discapacidad”. Siglo Cero: Revista Española sobre Discapacidad Intelectual, 42 (240): 50-78.

Martínez Martín, M. (2010): Los estudiantes con discapacidad en la Universidad de Burgos. Repositiorio Instituticional Universidad de Burgos. Tesis doctoral. Burgos: Universidad de Burgos.

Moneo, B. y Anaut, S. (2017): "Inclusión del alumnado con discapacidad en los estudios superiores. Ideas y actitudes del colectivo estudiantil”. Revista Española de Discapacidad, 5 (2): 129-148.

Moreno, J. F. et al. (2006): "Actitudes ante la discapacidad en el alumnado universitario matriculado en materias afines". Revista Iberoamericana de Educación, 40 (5): 1-12.

Moriña, A. et al. (2013): “El profesorado en la universidad ante el alumnado con discapacidad: ¿Tendiendo puentes o levantando muros?”. REDU. Revista de Docencia Universitaria, 11 (3): 423-442.

Moswela, E. y Mukhopadhyay, S. (2011): "Asking for too much? The voices of students with disabilities in Botswana". Disability \& Society, 26 (3): 307-319.

Novo-Corti, l. et al. (2011): "Análisis de las actitudes de los jóvenes universitarios hacia la discapacidad: un enfoque desde la teoría de la acción razonada". Revista Electrónica de Investigación y Evaluación Educativa, 17 (2).

Organización Mundial de la Salud (2001): International Classification of Functioning, Disability and Health: ICF, Génova: Organización Mundial de la Salud.

Polo, M. T. et al. (2011): "Study of the Attitudes of University Students Coming from Social Sciences and Psychology: Relevance of the Information and Contact with the Disabled". Universitas Psychologica, 10 (1): 113-123.

Reindal, S. M. (1995): "Some problems encountered by disabled students at the University of Oslo-whose responsibility?”. European Journal of Special Needs Education, 10 (3): 227-241.

Rodríguez-Martín, A. y Álvarez-Arregui, E. (2015): "Universidad y discapacidad: Actitudes del profesorado y de estudiantes". Perfiles educativos, 37 (147): 86-102.

Rodríguez-Martín, A. y Álvarez- Arregui, E. (2014): "Estudiantes con discapacidad en la Universidad. Un estudio sobre su inclusión". Revista Complutense de Educación, 25 (2): 457-479.

Rodríguez-Martín, A. y Álvarez-Arregui, E. (2013): "Development and validation of a scale to identify attitudes towards disability in Higher Education”. Psicothema, 25 (3): 370-376.

Salinas, M. et al. (2013): "La inclusión en la educación superior desde la voz de los estudiantes chilenos con discapacidad”. Revista Iberoamericana de Educación, 63: 77-98.

Sánchez Palomino, A. (2009): Integración educativa y social de los estudiantes con discapacidad en la Universidad de Almería. Almería: Universidad de Almería.

Suriá, R. et al. (2011): "Prejuicios entre los estudiantes hacia las personas con discapacidad: reflexiones a partir del caso de la Universidad de Alicante”. Alternativas. Cuadernos de Trabajo Social, 18: 75-90.

Suriá, R. (2011): "Análisis comparativo sobre las actitudes de los estudiantes hacia sus compañeros con discapacidad". Electronic Journal of Research in Educational Psychology, 9 (23): 197-216. 
Tinklin, T. y Hall, J. (1999): "Getting round obstacles: Disabled students' experiences in higher education in Scotland". Studies in Higher education, 24 (2): 183-194.

Verdugo, M. A. et al. (1995): "Actitudes sociales y profesionales hacia las personas con discapacidad: estrategias de evaluación e intervención”, en Verdugo, M. A. y Aguado, A. L. (coords.): Personas con discapacidad: perspectivas psicopedagógicas y rehabilitadoras. México: Siglo XXI. 